\title{
ESTRUCTURA METODOLÓGICA DE REVISTAS CON ALTO FACTOR DE IMPACTO SEGÚN EL CARÁCTER SUMATIVO Y FORMATIVO
}

\author{
George ARGOTA PÉREZ \\ Centro de Investigaciones Avanzadas y Formación Superior en Educación, \\ Salud y Medio Ambiente AMTAWI. Puno, Perú. \\ george.argota@gmail.com \\ Carlos CÓRDOVA SALAS \\ Universidad Nacional San Luis Gonzaga (UNICA). Ica, Perú. \\ Facultad de Agronomía. \\ carlos_r_1958@hotmail.com \\ Jorge MAGALLANES MAGALLANES \\ Universidad Nacional San Luis Gonzaga (UNICA). Ica, Perú. \\ Facultad de Agronomía. \\ jolmm999@hotmail.com

\begin{abstract}
Alicia IBARRA BOBER
Universidad Nacional San Luis Gonzaga (UNICA). Ica, Perú.

Facultad de Medicina Veterinaria y Zootecnia.

aibarraunica@gmail.com

Néstor GONZÁLEZ AEDO

Universidad Nacional San Luis Gonzaga (UNICA). Ica, Perú.

Facultad de Odontología.

olivergonzalesaedo@hmail.com
\end{abstract} \\ Gladys HUAMÁN ESPINOZA \\ Universidad Nacional San Luis Gonzaga (UNICA). Ica, Perú. \\ Facultad de Odontología. \\ rosarioesunica@gmail.com

\section{José IANNACONE} \\ Universidad Ricardo Palma. Lima, Perú. \\ jose.iannacone@urp.edu.pe
}

\section{ABSTRACT}

The aim of study was to evaluate the introductory methodological structure of journals with a high impact factor according to the summative and formative nature. The percentage of application was estimated according to the following character: summative, formative, comprehensive, scientific and check-evaluative for the last four volumes of five journals with different approaches (Biomedical Sciences, Environmental Sciences, Food y Nutrition, Pharmaceutical Sciences, Research y Reviews: Journal of Educational Studies). Each character was assigned the $25 \%$ score before the interpretation of being manifested in the published scientific articles excluding the check-evaluative character due to the fact that it was assumed as the evaluation form that the reviewers should follow, varying the same among the journals. 5\% was assigned among the methodological structures: introduction, 
materials and methods, results, discussion and conclusions. It was observed that the number of articles published among the numbers of the journals were: 18, 16, 16, 14 and 16 where the order of character corresponded to: scientific> comprehensive> summative $>$ formative. The character by methodological structures was: scientific and comprehensive (introduction), comprehensive (materials and methods), results (scientific, comprehensive), discussion (scientific, comprehensive, and summative) and conclusions (summative, formative). The evaluative perception was not uniform and the summative and formative character was limited in the introduction, and the identity of needs and the improvement on problems could be restricted.

\section{KEYWORDS}

Character - evaluation - impact factor - scientific publication

\section{METHODOLOGICAL STRUCTURE OF JOURNALS WITH HIGH IMPACT FACTOR ACCORDING TO THE SUMMARY AND TRAINING CHARACTER}

\section{RESUMEN}

El objetivo del estudio fue evaluar la estructura metodológica introductoria de revistas con alto factor de impacto según el carácter sumativo y formativo. Se estimó el porcentaje de aplicación según los siguientes caracteres: sumativo, formativo, comprensivo, científico y chequeo-evaluativo para los cuatro últimos volúmenes de cinco revistas con diferentes enfoques (Biomedical Sciences, Environmental Sciences, Food y Nutrition, Pharmaceutical Sciences, Research y Reviews: Journal of Educational Studies). A cada carácter se les asignó la puntuación del 25\% ante la interpretación de ser manifestado en los artículos científicos publicados excluyendo al carácter chequeo-evaluativo debido a, que se asumió como la ficha de evaluación que deben seguir los revisores variando la misma entre las revistas. Se asignó un 5\% entre las estructuras metodológicas: introducción, materiales y métodos, resultados, discusión y conclusiones. Se observó que, el número de artículos publicados entre los números de las revistas fueron: 18, 16, 16, 14 y 16 donde el orden del carácter correspondió a: científico > comprensivo > sumativo > formativo. El carácter por estructuras metodológicas fue: científico y comprensivo (introducción), comprensivo (materiales y métodos), resultados (científico, comprensivo), discusión (científico, comprensivo y sumativo) y conclusiones (sumativo, formativo). Fue no uniforme la percepción evaluativa y limitado el carácter sumativo y formativo en la introducción pudiendo restringirse la identidad de necesidades y la mejora sobre problemáticas.

\section{PALABRAS CLAVE}

Carácter - evaluación - factor de impacto - publicación científica

Recibido: $18 / 03 / 2020$

Aprobado: 29/05/2020 


\section{INTRODUCCIÓN}

ada año, se publica aproximadamente 2.5 millones de artículos científicos (El-Serag, 2012; Ware y Mabe, 2015) El mérito de los manuscritos científicos radica en su consistencia y coherente redacción (Auvinen, 2015; Onodera y Yoshikane, 2015; Van Steedam, 2016; Amjad et al., 2017;) siendo en primera instancia, la revisión exhaustiva de la literatura científica lo que condiciona hacia un conocimiento nuevo a comunicarse (Csiszar, 2016).

Cualquier análisis sobre las propuestas de manuscritos científicos, es una medida individual de la posible publicación (Rennie, 2016) de modo que, resulta necesario en términos formativos, valorar la calidad sobre lo que se propone, pues ello significaría un reflejo de la posible redacción personal y no basta, el desarrollo propiamente de la investigación por el contrario, asegurar la calidad intermedia y final de todo resultado a presentarse significará lo trascendental pero, demostrándose la reflexión basada en el conocimiento adquirido con esfuerzo (Blau, 2019).

Aunque cada estructura metodológica es independiente en sí misma, pero todas conforman un sistema funcional (Guerrero et al., 2019). La valoración acorde a la originalidad y relevancia de cualquier resultado (Sengupta et al., 2014) puede ser interpretada, no solo desde el título y el resumen sino, en la forma comunicativa del tipo de carácter que se expresa en la estructura metodológica de la introducción.

El objetivo del estudio fue evaluar la estructura metodológica introductoria de revistas con alto factor de impacto según el carácter sumativo y formativo.

\section{MATERIALES Y MÉTODOS}

Se seleccionó el número de artículos publicados en el año 2019 para cinco revistas científicas indexadas: Science of the Total Environment, Environmental Pollution, Chemosphere, Water Research y Ecotoxicology and Environmental Safety donde se analizó, el carácter de la información científica según las estructuras metodológicas: introducción, materiales y métodos (métodos), resultados, discusión (resultados y discusión) y conclusión (conclusiones).

Se interpretó el orden del carácter de las revistas (selección de artículos científicos mediante un muestreo probabilístico aleatorio $=25$ ) y luego, se valoró en cada estructura metodológica, la prevalencia del carácter el cual se clasificó en lo siguiente:

- Carácter científico: se emiten juicios de valor y toma de decisiones sobre el proceso investigativo.

- Carácter formativo: durante el proceso de investigación toda información es 
orientada a su propia mejora. Se identifican problemas, facilita alternativas, descubre obstáculos.

- Carácter sumativa: evalúa resultados durante un proceso (objetivos trazados), además, de las necesidades previas identificadas. Resulta de la adecuación de alcance a las necesidades sociales (beneficiarios).

- Carácter comprensivo: considera todo tipo de información (proveniente de los instrumentos de medición, aquellos formales e informales) para seleccionar los más adecuados.

Se utilizó el programa profesional versión libre Epidat 4.2 para la comparación del número de artículos entre las revistas científicas indexadas considerándose significativos los resultados cuando $\mathrm{p}<.05$.

Se consideró como aspectos éticos en el estudio lo siguiente:

1. Derechos

a) Consideración a participar en el estudio por los autores, una vez que los objetivos y los métodos hayan sido explicados.

b) Necesidad social para comunicar los resultados contribuyendo a la construcción de premisas en la redacción científica.

2. Deberes

a) Excluir toda posibilidad de engaños indebidos o, influencia.

\section{RESULTADOS Y DISCUSIÓN}

Se muestra el número de artículos por revistas científicas indexadas donde existió diferencias estadísticamente significativas: $\mathrm{p}<.05(\mathrm{~F}=514314.00 / \mathrm{P}=.00)$ (Tabla 1).

Tabla 1. Número de artículos científicos / revistas científicas indexadas.

\begin{tabular}{l|c|c}
\hline \multicolumn{1}{c}{ Revistas } & $\begin{array}{c}\text { Número de } \\
\text { artículos }\end{array}$ & $\begin{array}{c}\text { Homogeneidad de } \\
\text { grupos }\end{array}$ \\
\hline Science of the Total Environment & 1,174 & $\mathrm{a}$ \\
Environmental Pollution & 738 & $\mathrm{~b}$ \\
Chemosphere & 400 & $\mathrm{c}$ \\
Ecotoxicology and Environmental Safety & 264 & $\mathrm{~d}$ \\
Water Research & 154 & $\mathrm{e}$ \\
\hline
\end{tabular}

Letras (significan grupos homogéneos)

Se muestra la nomenclatura de las estructuras metodológicas de las revistas científicas indexadas siendo similar para las revistas Science of the Total Environment, Environmental Pollution, Chemosphere y Water Research (Tabla 2). 
Tabla 2. Nomenclatura de estructuras metodológicas / revistas científicas indexadas.

\begin{tabular}{l|c|c|c|c|c}
\hline \multicolumn{1}{c}{ Revistas } & $\mathrm{I}$ & $\mathrm{M}^{*}$ & $\mathrm{R}^{* *}$ & \multicolumn{1}{c}{$\mathrm{D}^{* *}$} & $\mathrm{C}^{* * *}$ \\
\hline Science of the Total Environment & $\mathrm{X}$ & $\mathrm{X}$ & $\mathrm{X}$ & $\mathrm{X}$ & $\mathrm{X}$ \\
Environmental Pollution * & & & & & \\
Chemosphere * $^{*}$ & & & & & \\
Water Research * $^{\text {Ecotoxicology and Environmental Safety,****** }}$ & $\mathrm{X}$ & $\mathrm{X}$ & & $\mathrm{X}$ & $\mathrm{X}$ \\
\hline
\end{tabular}

Leyenda: I (Introducción), M (Métodos), R (Resultados), D (Discusión), C (Conclusión)

* MyM (Materiales y Métodos), ${ }^{* *}$ RyD (Resultados y Discusión) *** (Conclusiones)

Se muestra el orden de carácter observado de acuerdo a, las estructuras metodológicas de las revistas científicas indexadas (Tabla 3).

Tabla 3. Orden de carácter / revistas científicas indexadas.

\begin{tabular}{l|c}
\hline \multicolumn{1}{c}{ Revistas } & Orden de carácter \\
\hline Science of the Total Environment & \\
Environmental Pollution & científico $>$ comprensivo $>$ \\
Chemosphere & sumativo $>$ formativo \\
Water Research & \\
Ecotoxicology and Environmental Safety & \\
\hline
\end{tabular}

Se muestra la identificación del carácter en cada una de las estructuras metodológicas de las revistas científicas indexadas donde el carácter sumativo y formativo, es poco valorado desde su propia introducción (Tabla 4).

Tabla 4. Identificación del carácter / estructuras metodológicas / revistas científicas indexadas.

\begin{tabular}{c|c|c|c|c|c}
\hline \multicolumn{2}{c}{ Introducción } & \multicolumn{1}{c}{$\begin{array}{c}\text { Materiales y } \\
\text { Métodos }\end{array}$} & \multicolumn{2}{c}{ Resultados } & \multicolumn{2}{c}{ Discusión } & \multicolumn{1}{c}{$\begin{array}{c}\text { Conclu- } \\
\text { siones }\end{array}$} \\
\hline Carácter & $\begin{array}{c}\text { Científico, } \\
\text { comprensivo }\end{array}$ & Comprensivo & $\begin{array}{c}\text { Científico, } \\
\text { comprensivo }\end{array}$ & $\begin{array}{c}\text { Científico, } \\
\text { comprensivo, } \\
\text { sumativo }\end{array}$ & $\begin{array}{c}\text { Sumativo, } \\
\text { formativo }\end{array}$ \\
\hline
\end{tabular}

Por lo general, la primera aceptación de todo manuscrito científico está en el llenado de ficha por el editor (a) (Salomón, 1957) y a pesar de, la variación entre los formatos de evaluación de las revistas, existe una similitud en sus criterios. Blau (2019) señaló que al menos seis criterios se admiten para mejorar la aceptabilidad del manuscrito los cuales pueden ser resumidos de la siguiente manera:

1. Claridad del propósito y calidad de la escritura.

2. Discusión y comprensión de la técnica. 
3. Justificación del enfoque.

4. Validación experimental de los modelos propuestos.

5. Novedad y exclusión de conclusiones obvias.

6. Presentación del trabajo bajo sus características de investigación y no, como informe de prueba.

Sin embargo, aún con el incremento significativo de los artículos científicos bajo el enfoque de coautorías para dimensionar la visibilidad colaborativa en las investigaciones científicas (Abt, 2007; Uddin et al., 2012; Bremmer et al., 2014) parece crítico que, la probable despreocupación en reconocer el tipo de carácter con el cual se redacta o considera la propuesta del manuscrito científico.

En este estudio se observó que, el rango de publicación de las revistas científicas indexadas estuvo entre 264 y 1174 . Aunque cada revista científica tiene su propio campo de investigación, área de investigación y temas de investigación, estas revistas guardan relación pudiendo valorarse que la mayor probabilidad para publicar un manuscrito se establece en el siguiente orden: Science of the Total Environment $>$ Environmental Pollution $>$ Chemosphere $>$ Ecotoxicology and Environmental Safety $>$ Water Research.

Todas las revistas científicas indexadas presentaron similar estructura metodológica con relación a la introducción pero varió su nomenclatura entre declarar si es materiales y métodos o métodos así como, si es resultados y discusión o considerarse por separado. Se observó cómo detalle considerar en singular o plural la conclusión o conclusiones. En este último aspecto, quizás sea relativo, pues si es referido al objetivo, puede ser valorado el número gramatical de singular si se refiere al objetivo pero, si lo que se desea es el análisis de las variables dentro del objetivo, entonces se valoró como plural.

El carácter científico fue el que prevaleció y ello significó que toda propuesta de manuscrito pretendido a investigar debió mostrar la contrastación de las variables desde lo gnoseológico y epistemológico para denotar incremento del conocimiento según lo hallado pero poco se valoró en la redacción de los propios artículos científicos, el análisis para su continuidad temática. En este sentido, podría realizarse un análisis correlación para corroborarlo. Del mismo modo, no parece lógico a partir de, la interpretación sobre la estructura de los resultados que el carácter sea correcto, pues si en la identificación de materiales y métodos (métodos) correspondió al carácter comprensivo, entonces con este carácter debe iniciarse para la estructura metodológica de los resultados. La calificación como proceso de aprendizaje (Lee y Lee, 2020) requiere de conocimientos no solo demostrados sino, adquirirse donde se necesita de forma permanente, evaluar los resultados durante un proceso y adecuar las posibles necesidades de requerimiento hacia sus beneficiarios (reconocimiento de valor sumativo).

Cuando se realiza la discusión del comportamiento sobre los hallazgos en ocasiones puede que las variables se comporten como "latentes", es decir, solo resulta un fenómeno de interés teórico (Scholderer y Balderjahn, 2006) y al no ser 
observable directamente, requiere ser evaluado (construcción y medidas: items e indicadores) mediante un carácter sumativo y posteriormente, formativo.

Cualquier estrategia que se redacte y genere una evaluación, impulsa el aprendizaje en un contexto de reconocimiento que poder ser sumativo (Nyoni y Botma, 2017) pero debe estar basada en su condición de reflexiva (Blau, 2019). En este estudio, la transición desde el carácter sumativo al formativo resultó confusa, pues si en la introducción es donde se expresa el objetivo a medirse y su carácter correspondió a científico y comprensivo, entonces como las conclusiones presentan otros tipos de carácter (sumativo y formativo).

Diamantopoulos, Riefler y Roth (2008) y Edwards (2011) señalan que, una de las razones más trascendentales para la medición de las variables cuando son multidimensionales es su construcción formativa. Romero et al., (2014) indica que, la evaluación formativa al ser integrada en el proceso de enseñanza/aprendizaje implica, la generación de opiniones retroalimentadas y es en tal sentido, la razón durante la escritura de algunos artículos científicos que debe plasmarse independientemente que sea en su modalidad de opinión o no.

La principal limitación del estudio fue, realizar comparaciones con revistas de perfil social.

Se concluyó que, la percepción evaluativa fue no uniforme, además, de limitado el carácter sumativo y formativo en la introducción donde se restringió la identidad de necesidades y la mejora sobre problemáticas pretendidas a investigar.

\section{REFERENCIAS BIBLIOGRÁFICAS}

Abt, H.A. (2007). The future of single-authored papers. Scientometrics, 73, 353358.

Amjad, T., Ding, Y., Xu, J., Zhang, C., Daud, A., Tang, J. y Song, M. (2017). Standing on the shoulders of giants. Journal of Informetrics, 11, 307-323.

Auvinen, A. (2015). How do I write a scientific article-advice to a young researcher. Duodecim, 131, 1460-1466.

Blau, P.J. (2019). An editor's perspective on the quality and content of wear research manuscripts. Wear, 426-427, 1384-1390.

Bremner, S., Pierson, S.A., Jones, R. y Bhatia, V. (2014). Task design and interaction in collaborative writing: The students' story. Business and Professional Communication Quarterly, 77, 150-168.

Csiszar A. (2016). Peer review troubled from the start. Nature, 532, 306-308.

Diamantopoulos, A., Riefler, P. y Roth, K.P. (2008). Advancing formative measurement models. Journal of Business Research, 61, 1203-1218. 
Edwards, J.R. (2001). The fallacy of formative measurement. Organizational Research Methods, 14, 370-88.

El-Serag, H.B. (2012). Writing and publishing scientific papers. Gastroenterology; $142,197-200$.

Guerrero, S.J.D.T., Chicharro, F.P.R., Serrano, G.J., Menendez, D.V. y Castellanos, B.M.E. (2019). A proposal for a recommender system of scientific relevance. Procedia Computer Science, 162, 199-206.

Lee, S.W. y Lee, E.A. (2020). Teacher qualification matters: The association between cumulative teacher qualification and students' educational attainment. International Journal of Educational Development, 77, 1-10.

Nyoni, C.N. y Botma, Y. (2017). Aligning summative clinical examination with competence-based curriculum: Midwifery educators experiences in Lesotho. International Journal of Africa Nursing Sciences, 7, 11-17.

Onodera, N. y Yoshikane, F. (2015). Factors affecting citation rates of research articles. Journal of the Association for Information Science and Technology, 66, 739-764.

Rennie, D. (2016). Let's make peer review scientific. Nature, 535, 31-33.

Romero, M.R., Fraile, A.A., López, P.V.M. y Castejón, O.F.J. (2014). The relationship between formative assessment systems, academic performance and teacher and student workloads in higher education. Infancia \& Aprendizaje, 37, 310341.

Salomon, G (1957). Editorial. Wear, 1, 1-4.

Scholderer, J. y Balderjahn, I. (2006). Was unterscheidet harte und weiche Strukturgleichungsmodelle nun wirklich? Marketing ZFP - Journal of Research and Management, 28, 57-70.

Sengupta, S., Shukla, D., Ramulu, P., Natarajan, S. y Biswas, J. (2014). Publish or perish: the art of scientific writing. Indian Journal of Ophthalmology, 62, 10891093.

Uddin, S., Hossain, L., Abbasi, A. y Rasmussen, K. (2012). Trend and efficiency analysis of co-authorship network. Scientometrics, 90, 687-699.

Van Steendam, E. (2016). Editorial: forms of collaboration in writing. Journal of Writing Research, 8, 183-204.

Ware, M. y Mabe, M. (2015). The STM Report an Overview of Scientific and Scholarly Journal Publishing. Fourth Edition (The Hague, Netherlands). University of Nebraska-Lincoln, USA. pp. 1-181. 